\title{
Lenalidomide following fludarabine and rituximab in previously untreated CLL
}

Chaitra Ujjani ${ }^{*}$, Aneel Paulus², Edmund Gehan ${ }^{3}$, Saad Jamshed ${ }^{4}$, Tingting Zhuang ${ }^{5}$, Kasyapa Chitta², Gaihua Zhang ${ }^{2}$, Yan Asmann ${ }^{6}$, Jeanette Crawford ${ }^{1}$ and Bruce D. Cheson ${ }^{1}$

${ }^{*}$ Correspondence: csu@georgetown.edu

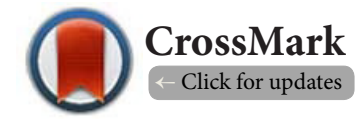

'Department of Hematology and Oncology, Lombardi Comprehensive Cancer Center, Georgetown University Hospital, 3800 Reservoir Rd NW, Washington, DC, USA.

${ }^{2}$ Department of Cancer Biology, Mayo Clinic, 4500 San Pablo Road South, Jacksonville, FL, USA.

${ }^{3}$ Department of Biostatistics, Bioinformatics and Biomathematics, Georgetown University Hospital, 3800 Reservoir Rd NW, Washington, DC, USA.

${ }^{4}$ Department of Hematology and Oncology, Rochester General Hospital, 1425 Portland Ave Rochester, NY 14621, USA.

${ }^{5}$ Department of Biostatistics, University at Buffalo, 711 Kimball Tower, Buffalo, NY 14214, USA.

${ }^{6}$ Department of Health Sciences Research, Mayo Clinic, 4500 San Pablo Road South, Jacksonville, FL, USA.

\begin{abstract}
Fludarabine-based regimens have overall response rates (ORR) of 90-95\% in untreated chronic lymphocytic leukemia (CLL), but almost all patients inevitably relapse. Given the promising activity of lenalidomide in relapsed/refractory CLL, we studied the combination of fludarabine and rituximab (FR) followed by lenalidomide in untreated disease $(\mathrm{n}=22)$. Patients received three cycles $(\mathrm{C})$ of $\mathrm{FR}\left(\mathrm{F} 25 \mathrm{mg} / \mathrm{m}^{2}\right.$ days (D) $\left.1-5, \mathrm{R} 375 \mathrm{mg} / \mathrm{m}^{2} \mathrm{D} 1\right)$. Those who responded received three more cycles of FR followed by three cycles of lenalidomide; whereas patients with stable or progressive disease switched to lenalidomide (5 mg C1D1-21, $10 \mathrm{mg}$ in subsequent cycles). After three cycles of lenalidomide, patients with a response or stable disease received another three cycles, whereas those who progressed were removed. The ORR with FR was 77\% (complete response (CR) 23\%). With lenalidomide $(\mathrm{n}=15)$, three converted from a partial response to $\mathrm{CR}$, increasing the $\mathrm{CR}$ rate to $36 \%$. The most common > grade 3 toxicity was neutropenia (59\%). The median progression-free survival was 38.5 months $(95 \%$ CI: 32.0, 53.9) and the median overall survival had not yet been reached at the time of analysis. The study demonstrated the feasibility of FR-L when administered in a sequential manner.
\end{abstract}

Keywords: Chronic lymphocytic leukemia (CLL), immunomodulatory agents, ludarabine, lenalidomide

\section{Introduction}

Chronic lymphocytic leukemia (CLL) is the most common form of adult leukemia in the western world, affecting about 15,000 new patients each year in the United States [1]. Whereas most patients do not require therapy at diagnosis, CLL often progresses and treatment is indicated for signs or symptoms of the disease. Standard front-line regimens at the time of initiation of this study included fludarabine and rituximab (FR) with or without cyclophosphamide. The Cancer and Leukemia Group B (CALGB) 9712 study demonstrated an overall response rate (ORR) of $90 \%$ [complete response (CR): $47 \%$ ] and a median overall survival (OS) of 91 months with concurrent FR [2,3]. Based on encouraging data with fludarabine and cyclophosphamide (C) and the potential added benefit of rituximab, both the MD
Anderson Cancer Center (MDACC) and the German CLL Study Group evaluated the triplet, FCR. While the single-center data from MDACC demonstrated an impressive ORR of $95 \%$ and CR of 72\% [4]. The multi-institutional German data was more consistent with the previously reported CALGB 9712 data with an ORR of $90 \%$ (CR 44\%) [5].

Despite the initial efficacy with fludarabine-based regimens, almost all patients inevitably relapse, and additional treatment options must be considered. Immunomodulatory agents have made an impact in various hematologic malignancies, especially those of B-cell origin. In a phase I/II study of thalidomide and fludarabine in previously untreated CLL, Chanan-Khan et al., reported an ORR of 100\% [6]. This obse-rvation led to the investigation of lenalidomide in CLL, which as a single agent in 
Ujjani et al. Hematology and Leukemia 2015,

the relapsed/refractory setting, demonstrated an ORR of $58 \%$ with a $C R$ rate of $18 \%[7,8]$. A subsequent study by Ferrajoli et al., validated the clinical effectiveness of lenalidomide in CLL [9] launching a series of clinical trials investigating this agent both in combination with monoclonal antibodies as well as a mediator to enhance response to estab-lished chemoimmunotherapeutic approaches.

The rationale for combining lenalidomide with immunotherapy was to capitalize upon its ability to modulate the host T- and NK-cell immune response. However, the premise behind the addition of lenalidomide to chemotherapy was to undermine the tumor microenvironment dependent prosurvival pathways through inhibition of critical cytokines (IL-6, VEGF, PDGF), thus increasing the cytotoxic effects of the chemotherapy. Brown et al., first investigated the combination of concurrent lenalidomide and FR in previously untreated patients, reporting considerable toxicity, including prolonged myelosuppression, severe rash, and tumor flare reactions [10]. Many patients were unable to complete therapy and concurrent lenalidomide with FR was deemed intolerable. The Revlirit CLL5 AGMT (Arbeitsgemeinschaft Medikamentöse Tumortherapie) study also evaluated the combination in treatment naïve patients $[11,12]$. Impressive activity was noted (ORR of 96\%, median progression-free survival (PFS) 46 months), however, many patients experienced significant skin toxicity and neutropenia. In a recent phase I/II study, Flinn et al., noted better tolerability but lower efficacy than previously reported: ORR 63\% (CR 11\%) [13]. Given the considerable toxicity of concurrent lenalidomide and $F R$, we investigated the novel strategy of lenalidomide as a maintenance therapy following FR induction in order to determine its impact on clinical responses. Correlative studies were designed to explore molecularly distinct patient groups that may demonstrate enhanced responsiveness to this approach.

\section{Methods}

The study was approved by the ethics committee and institutional review board at the Lombardi Comprehensive Cancer Center at Georgetown University Hospital. All patients provided written informed consent. The study was sponsored by Lombardi CCC and Celgene Corporation. It was open from January 2008 to January 2011, and was the only front-line CLL study available at our institution. This study was one of the first to be open as part of our CLL program.

\section{Patients}

Patients >18-years-old with previously untreated CLL requiring therapy as per the NCI Working Group Guidelines for CLL [14] were eligible. In addition, patients must have had an ECOG performance status of $\leq 2$ and an absolute neutrophil count $\geq 1.0 \times 10^{9} / \mathrm{L}$, platelet count $\geq 75 \times 10^{9} / \mathrm{L}$, serum creatinine $\leq 1.5 \mathrm{mg} /$ $\mathrm{dL}$, total bilirubin $\leq 1.5 \mathrm{mg} / \mathrm{dL}$, and AST and ALT $\leq 2 x$ upper limit of normal (ULN) or $\leq 5 \times$ ULN if related to CLL. All patients had to be able to take aspirin for prophylaxis. Patients intolerant to aspirin could use warfarin or low molecular weight heparin. Patients with a history of a thromboembolic event received low molecular weight heparin with lenalidomide.

Exclusion criteria included the use of any other experimental therapy within 28 days of enrollment, concurrent use of other anti-cancer therapies, pregnancy, lactation, HIV, active hepatitis A or B, active hemolysis, severe cardiac conditions including myocardial infarction within 6 months, New York Hospital Association Class III/IV heart failure, arrhythmias, or any serious illness that prevented the patient from signing the consent form, placed the patient at unacceptable risk due to participation, or confounded the ability to interpret trial data.

\section{Treatment}

In this phase II, single-center, open-label study, patients received induction with fludarabine $25 \mathrm{mg} / \mathrm{m}^{2}$ intravenously on day (D) $1-5$ and rituximab $375 \mathrm{mg} / \mathrm{m}^{2}$ intravenously on D1 of each 28-day cycle, as in the CALGB 9712 dosing schedule. Patients at high risk for tumor lysis syndrome, defined as a white blood cell count $>75,000 / \mathrm{mm}^{3}$ or high tumor burden, received oral allopurinol $300 \mathrm{mg}$ daily on D-2 through D14 and intravenous normal saline on D1, 2 of Cycle (C) 1. Patients received prophylactic oral sulfamethoxazole/trimethoprim 160/800 mg twice daily on Mondays, Wednesdays, and Fridays and oral acyclovir $400 \mathrm{mg}$ twice daily continuously for antimicrobial prophylaxis at the discretion of the treating physician. The use of granulocyte colony stimulating factor was permitted for prophylaxis at the discretion of the treating physician in patients who experienced Grade 3 neutropenia with fever or Grade 4 neutropenia with or without fever.

Patients who achieved a partial response (PR) or CR after C3 of $F R$ received three additional cycles of $F R$. On completion of six cycles of $F R$, oral lenalidomide monotherapy was initiated within 28-56 days at $5 \mathrm{mg}$ daily D1-21 of each 28-day cycle. Patients who demonstrated stable or progressive disease (PD) after completing $\mathrm{C} 3$ of $\mathrm{FR}$ received lenalidomide at the same dose and schedule. All patients received aspirin $81 \mathrm{mg}$ daily with initiation of lenalidomide. Patients intolerant to aspirin were allowed to use warfarin or low molecular weight heparin. If tolerability permitted, the lenalidomide dose was increased to $10 \mathrm{mg}$ at $\mathrm{C} 2$ and for subsequent cycles. Patients received a maximum of six cycles of lenalidomide. Dose modifications and delays were permitted for toxicity.

\section{Assessments}

Response and progression were evaluated as per the $\mathrm{NCl}$ Working Group Criteria for Response in CLL [14]. Adverse events were evaluated as per the $\mathrm{NCl}$ Criteria for Adverse Events, version 3.0. Response assessments during induction and lenalidomide monotherapy were performed after every cycle by physical exam and laboratory blood tests. Imaging with computed tomography (CT) was performed prior to treatment, before initiating lenalidomide, and after completion of therapy. $\mathrm{CT}$ imaging could be performed after $\mathrm{C} 3$ of $\mathrm{FR}$ and after $\mathrm{C} 3$ 
of lenalidomide monotherapy if clinically indicated. Bone marrow biopsies were performed prior to treatment, prior to initiation of lenalidomide, at the completion of all therapy if the patient achieved a complete response by imaging, and whenever clinically indicated. Minimum residual disease assessment of bone marrow specimens at restaging time points were performed via real-time quantitative PCR for immunoglobulin heavy chain, 2-color flow cytometry, and immunohistochemistry, along with CLL fluorescence in situ hybridization (FISH) analysis. Cytogenetic and FISH studies were submitted to Quest Diagnositics for analysis. CD38 and Zap-70 status were assessed by flow cytometry and defined as positive for ZAP- $70>20 \%$ and CD38 > 30\%.

\section{Statistical considerations}

The primary endpoints of the study were (1) CR rate to FR and (2) $C R$ rate to $F R$ followed by the addition of lenalidomide. Secondary endpoints included ORR, PFS, safety, and the change in minimal residual disease with the addition of lenali-domide. This study used a Simon two-stage design, with a target enrollment of 41 patients. Twenty-one patients were to be entered in Stage 1. If the $C R$ rate was $>48 \%$, then the study would proceed to Stage 2 where another 20 patients would be enrolled. If the $C R$ rate was $<48 \%$, the study would be terminated at the end of Stage 1.

Descriptive statistical methods and confidence intervals (CI) were used to characterize the endpoints in the study. The PFS curve and overall survival (OS) curve were estimated by Kaplan-Meier method. The PFS was measured from the date of first treatment to the date of progression, last contact or death due to any cause. The OS was measured from the date of first treatment to the date of death or last contact.

\section{Correlative analyses}

RNA-Sequencing (RNA-Seq) was performed on tumor cells from CLL patients. Peripheral blood mononuclear cells were collected from patients $(n=15)$ on D0, prior to FR therapy. Cells were prepped for RNA purification using the Qiagen miRNeasy mini Kit (Qiagen, Venlo, the Netherlands) and Qiagen Qiacube (Qiagen, Venlo, the Netherlands) instrument. Only samples with RIN values $>7$ were included in the subsequent analyses. Transcriptome sequencing was performed at the Mayo Medical Genome Facility in Jacksonville, FL. The Illumina TruSeq RNA libraries from samples were sequenced using the paired-end protocol with a read length of 50-bp. A detailed description of the analyses is provided in the Supplementary Data section.

\section{Results \\ Patient characteristics}

Twenty-two patients were enrolled between January 2008 and January 2011. Baseline characteristics for the 22 patients are summarized in Table 1. The median age at enrollment was 60.5 years. The majority of patients were male, had Stage I/II disease, with low $\beta-2$ microglobulin levels and overexpression of
Table 1. Patient characteristics.

\begin{tabular}{|c|c|}
\hline Characteristics & No. of pts (\%) \\
\hline \multicolumn{2}{|l|}{ Sex } \\
\hline Male & $15(68 \%)$ \\
\hline Female & $7(32 \%)$ \\
\hline \multicolumn{2}{|l|}{ Age (years) } \\
\hline Median & 60.5 \\
\hline Range & $41-78$ \\
\hline \multicolumn{2}{|l|}{ ECOG performance status } \\
\hline 0 & $15(68 \%)$ \\
\hline 1 & $6(27 \%)$ \\
\hline 2 & $1(5 \%)$ \\
\hline \multicolumn{2}{|l|}{ Rai stage } \\
\hline $\mathrm{I} / \mathrm{II}$ & $16(73 \%)$ \\
\hline III/IV & $6(27 V)$ \\
\hline \multicolumn{2}{|l|}{ Cytogenetics by FISH } \\
\hline del 13q (sole abnormality) & $4(18 \%)$ \\
\hline Normal & $4(18 \%)$ \\
\hline Complex ${ }^{*}$ & $2(10 \%)$ \\
\hline del 11q & $7(32 \%)$ \\
\hline del 17p & $3(14 \%)$ \\
\hline \multicolumn{2}{|l|}{ B-2 microglobulin } \\
\hline$<4 \mathrm{mg} / \mathrm{L}$ & $18(82 \%)$ \\
\hline$>4 \mathrm{mg} / \mathrm{L}$ & $4(18 \%)$ \\
\hline CD38 expression $>30 \%$ & $16(73 \%)$ \\
\hline Zap-70 expression $>20 \%$ & $8(36 \%)$ \\
\hline
\end{tabular}

CD38. All patients were included in the intent-to-treat analysis.

\section{Efficacy}

The ORR with FR induction was $77.2 \%$ and the CR rate was $22.7 \%$. With the addition of lenalidomide in fifteen patients, three improved their response from a PR to $C R$, increasing the $C R$ rate to $36.4 \%$. Two of these patients experienced resolution of lymphadenopathy while one patient achieved eradication of minimal residual disease. The median time to response was 7 months (Range: 6-8 months). This time point correlates with first formal response assessment for the trial; however, many patients showed evidence of clinical response prior to this time point. Responses varied with cytogenetic risk group. The highest response rates were noted in those with del13q (ORR 100\%, CR 50\%) followed by those with del $11 q$ (ORR $86 \%$, CR 57\%). Of the three patients who did not respond to FR, FISH revealed del 17p in two, along with other cytogenetic abnormalities, and one patient with combined trisomy 12, del 11q, and abnormalities of 13q. Lenalidomide 
Ujjani et al. Hematology and Leukemia 2015,

was administered to two of these patients, but failed to induce a response. The third patient was removed from the study due to rapidly progressive disease. The predefined endpoint at the end of Stage 1 ( $C R>48 \%$ ) was not reached, thus the study did not proceed to Stage 2 .

The median PFS was 38.5 months with 95\% confidence interval of $(32.0,53.9)$. Figure 1 presents the PFS curve with $95 \%$ confidence intervals. Two patients who developed malignancies while on study were censored in the PFS. One patient developed breast cancer which was diagnosed on restaging imaging after completing 6 cycles of FR. Although she had achieved a partial response with induction, she was subsequently removed from study and did not receive lenalidomide. Of note, she had del 17p with evidence of CLL progression at twelve months after completing FR. The second patient, who elected not to receive lenalidomide, developed Hodgkin's lymphoma four months after completing FR. The median OS had not yet been reached at the time of analysis. Figure 2 presents the OS curve with $95 \%$ confidence intervals. Six deaths occurred on the study; one patient died after C2 from bacteremia and associated septic shock, three patients died of complications related to progressive disease, and two patients died of causes not available to us as they were followed at other centers.

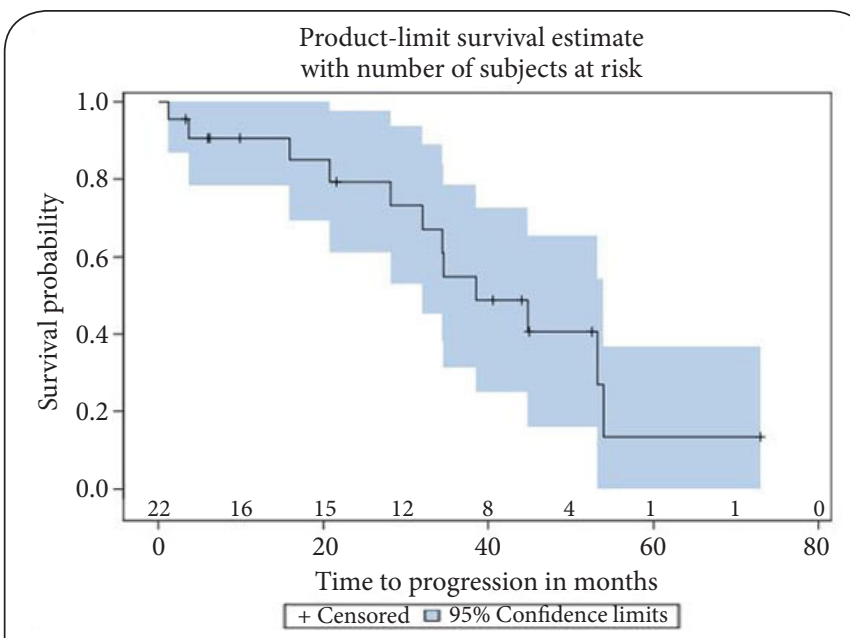

Figure 1. Progression-free survival. Kaplan-Meier curve of progression-free survival in months.

\section{Toxicity}

Table 2 includes hematologic toxicities experienced during FR and lenalidomide, respectively. The most common > grade 3 adverse event in either portion of the study was neutropenia, which occurred in $59 \%$ of patients during FR and $27 \%$ of patients during lenalidomide. Less frequent $>$ grade 3 adverse events with FR included thrombocytopenia, neutropenic fever, and anemia. One patient developed grade 3 thrombocytopenia with lenalidomide.

The most common non-hematologic adverse event during

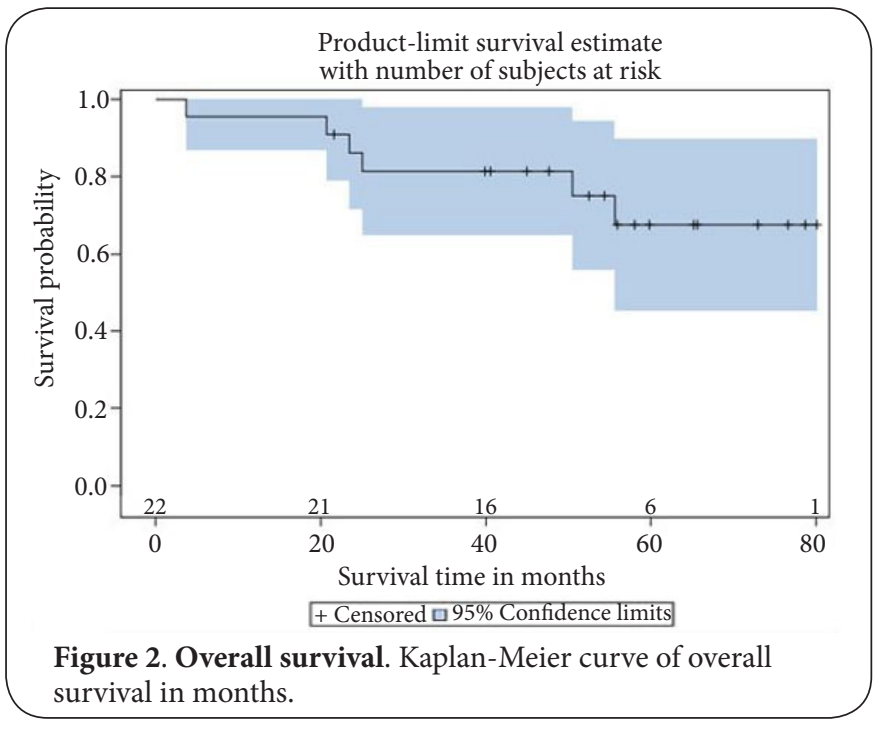

Table 2. Hematologic adverse events.

\begin{tabular}{lll}
\hline \multicolumn{3}{l}{ Hematologic adverse events with FR } \\
\hline CTC term & Grade 1/2 (n) & Grade 3/4 (n) \\
\hline Anemia & 12 & 4 \\
Neutropenia & 2 & 13 \\
Febrile neutropenia & 0 & 2 \\
Thrombocytopenia & 2 & 4 \\
\hline Hematologic adverse events with lenalidomide \\
\hline CTC term & Grade 1/2 (n) & Grade 3/4 (n) \\
\hline Anemia & 3 & 0 \\
Neutropenia & 4 & 4 \\
Thrombocytopenia & 6 & 1 \\
\hline
\end{tabular}

FR was fatigue, primarily grade $1-2$, which occurred in $36 \%$ of patients. Two patients developed >grade 3 infection, one of whom suffered a fatal septic shock secondary to a bacteremia in the setting of severe neutropenia during C2. There were no other grade 5 adverse events noted on study. Other grade 3-4 toxicities included renal failure $(n=2)$, catheter-related line thrombosis $(n=1)$, chylothorax $(n=1)$, and elevated hepatic transaminitis $(n=1)$.

The most common reasons for early withdrawal from the trial were rash and fatigue due to lenalidomide. Five patients developed a rash, the most severe of which was grade 3 in one patient and resulted in withdrawal from the study. Six patients developed grade 1-2 fatigue while on lenalidomide. There were two episodes of infection, including one grade 3 pneumonia. One patient developed severe hepatotoxicity, prompting withdrawal from the study. Figure 3 provides a summary diagram reviewing the distribution of patients throughout the treatment algorithm and indications for withdrawal from study. 


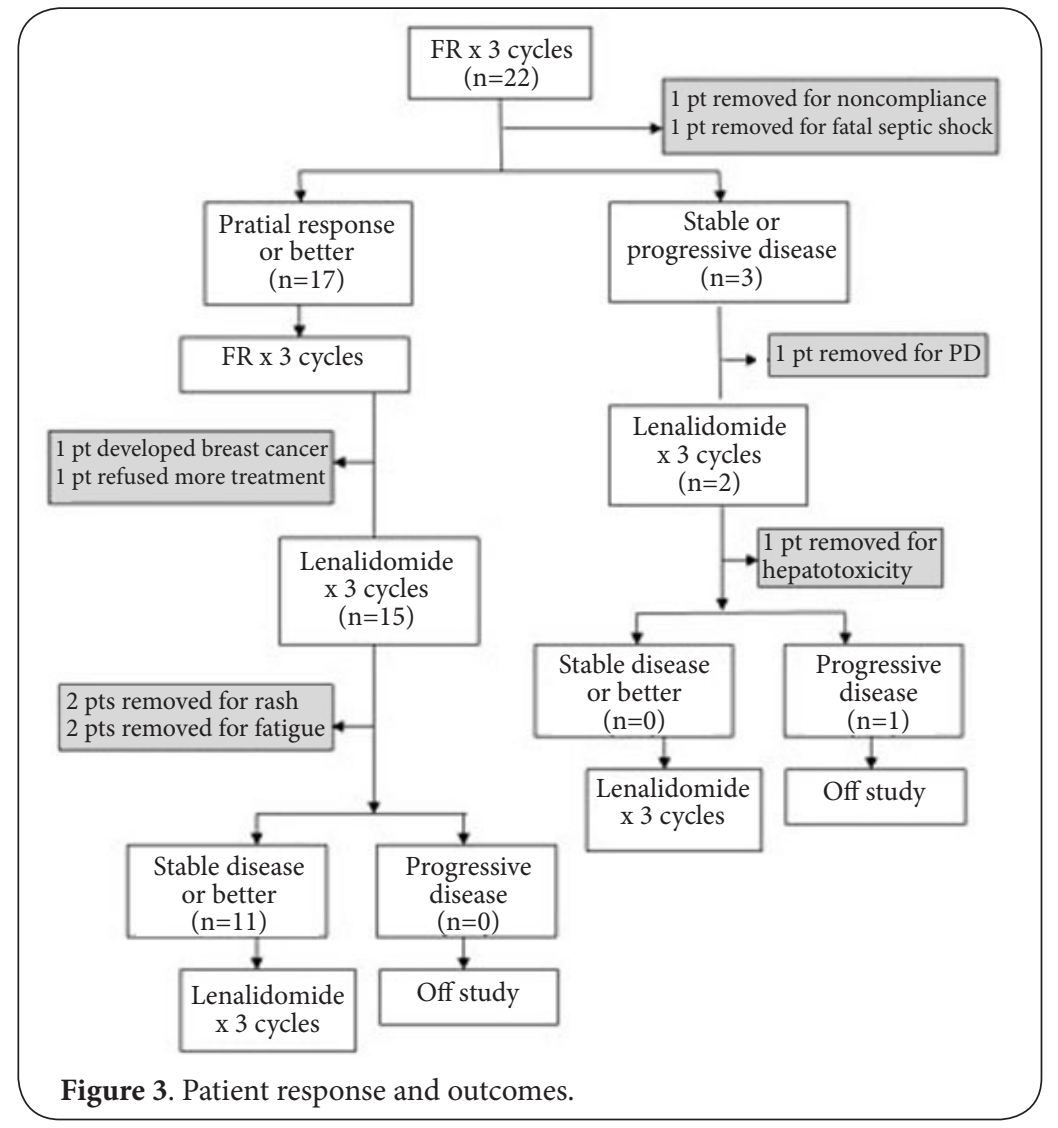

\section{Dose modifications}

Fifty percent of patients completed 12 cycles of therapy 6 cycles of FR and 6 cycles of lenalidomide). Patients completed a median of 6 cycles of FR (Range 2-6). Four patients required a $20 \%$ dose reduction of fludarabine; three required a dose reduction by $\mathrm{C} 4$, and one required a dose reduction by C6. Based on the original treatment algorithm of 6 cycles of induction, patients received $83 \%$ of the expected dose intensity of fludarabine. Patients completed a median of 4.5 cycles of lenalidomide (Range 0-6). The median tolerated dose of lenalidomide was $5 \mathrm{mg}$ (Range: $5 \mathrm{mg}$ every other day-10 mg daily). Of the seventeen patients who received at least one dose of lenalidomide, one developed grade 4 hepatotoxicity at a dose of $10 \mathrm{mg}$ daily, prompting withdrawal after $\mathrm{C} 3$. Another patient withdrew from study during $\mathrm{C} 3$ due to severe rash, despite dose reduction. Based on the initial schedule of 6 cycles of lenalidomide, including dose escalation to $10 \mathrm{mg}$ daily with C8, patients received $33 \%$ of the expected dose intensity lenalidomide.

\section{Correlative analysis}

We examined the transcriptome of CLL patients $(n=15)$ to delineate changes in the genetic circuitry and whether they corresponded with response and duration of remission. We first determined differentially expressed genes in All-CR patients, defined as all patients who achieved $C R$ with or without addition of lenalidomide to FR (All-CR samples $n=6$ ) versus Non- $C R$ patients ( $P R+P D$ samples, $n=9$ ). On unsupervised hierarchical clustering analysis, the majority of samples clustered together according to respective patients clinical response (Figure 4). ANOVA (2-fold change cutoff) between the All-CR and Non-CR groups demonstrated 33 genes and 49 genes were upregulated and downregulated, respectively, in All-CR samples relative to Non-CR samples. The differentially expressed genes are presented in Supplementary Table $\mathbf{S 1}$.

We also examined the transcriptome differences between patients who relapsed early (PFS $<2$ years, $n=5$ ), compared to those patients who had delayed relapse ( $P F S>2$ years, $n=10$ ). Clinical characteristics indicated that early relapse patients belonged to the Non-CR group and carried a more complex cytogenetic/mutation profile than late relapsing patients. Samples from the early and late relapse groups clustered according to their respective relapse pattern on a principal component analysis (Figure 5A) and hierarchical clustering analysis (Figure 5B). Transcriptome profiling demonstrated 159 genes that were differentially altered between patients who had experienced an early or a late relapse (cutoff at 2 -fold, $p<0.05$ ), wherein 121 genes were upregulated and 38 genes were downregulated in early relapsing patients (see Supplementary Table $\mathbf{S} 2$ for list of genes). Numerous tumor supporting genes such as BCL9 [15] (2.6-fold increase), CD38 (3.19-fold increase), and KLF5 (2.3-fold increase) [16] and 


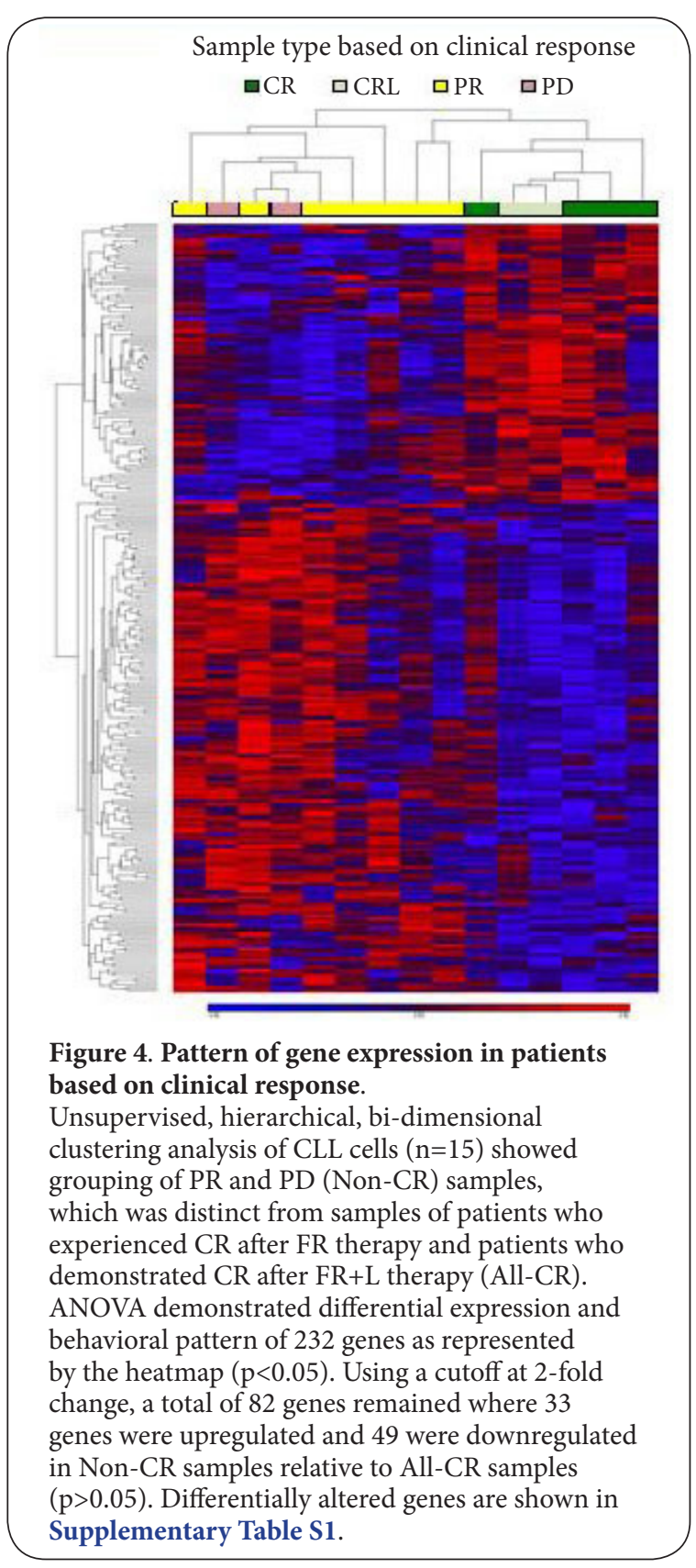

tumor suppressors such as TP73 (3.8-fold decrease) were significantly altered in early relapsers. We also found Signaling Lymphocytic Activation Molecule Family Member 1 (SLAMF1) transmembrane receptor levels to be 5 -fold decreased; whose reduced expression has been reported to correlate with shorter time to treatment and poor prognosis $[17,18]$. Although limited by availability and sample size, the results from our correlative analyses provide a framework for further hypothesis generation and will be validated in larger datasets.

\section{Discussion and conclusion}

At the time of study conception, fludarabine-based chemoim-

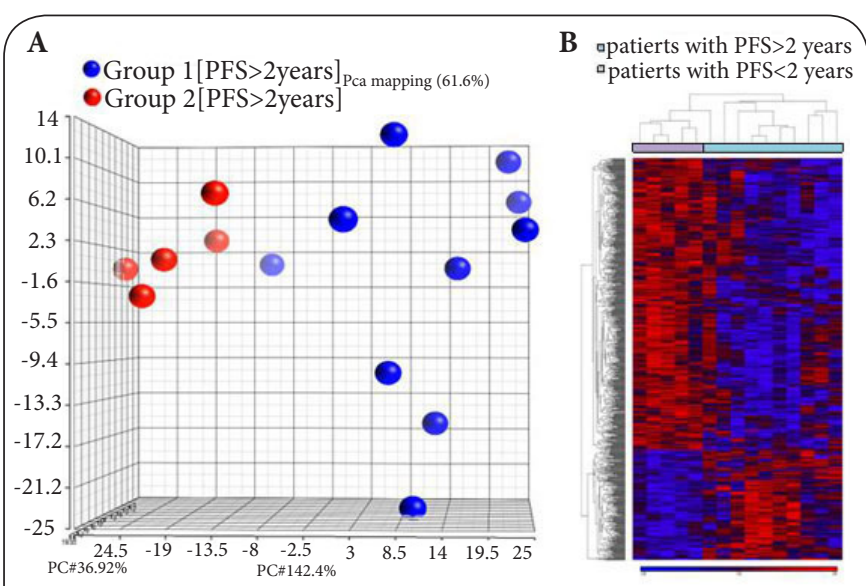

Figure 5. Principle component and gene expression analysis of samples according to PFS.

Sequenced samples from patients with PFS $<2$ years (early relapsing patients) was compared against patients whose PFS $>2$ years (late relapsing patients). (A) Principal component analysis (PCA) plot showing transcriptome differences between samples from patients who had a PFS $>2$ years (blue circles) compared to samples from patients who had a PFS $<2$ years (red circles). The PCA plot captures the variance in a dataset in terms of principal components and displays the most significant of these on the $\mathrm{x}, \mathrm{y}$, and $\mathrm{z}$-axes. The percentages of the total variation that are accounted for by the $1^{\text {st }}, 2^{\text {nd }}$, and $3^{\text {rd }}$ principal components are shown on the $\mathrm{x}^{-}, \mathrm{y}^{-}$ and z-axes labels. (B) Gene expression analysis and ANOVA revealed a total of 621 genes were differentially expressed. A total of 159 genes were differentially altered between early relapsing patients compared to late relapsing patients (2-fold change cutoff). In early relapsing patients, 121 genes were upregulated and 38 were downregulated $>2$ fold relative to late relapsing patients. Scale color of red indicates upregulation of gene, whereas blue indicates downregulation. Differentially altered genes are shown in Supplementary Table S2.

munotherapy was the standard of care for previously untreated CLL. While concurrent FR and lenalidomide is associated with significant toxicity, lenalidomide as maintenance therapy following FR induction has not yet been reported. In this study, the addition of lenalidomide in a sequential manner allowed for better tolerability and less toxicity than prior data with concurrent regimens of FRL [12]. The most common side effect was fatigue, which was primarily grade 1-2 in severity. While grade 3-4 neutropenia occurred in nearly $60 \%$ of patients, the incidence of severe infections was less than $20 \%$. Given the one patient who succumbed to fatal bacterial sepsis, the high incidence of severe neutropenia with FR is concerning and remains an area for improvement for future regimens.

Unfortunately, our study was unable to confirm the efficacy of prior reports of FR in previously untreated CLL. In the CALGB 9712 trial an ORR of $90 \%$ was noted with concurrent FR [2]. Even with the addition of lenalidomide to the exact same regimen, our CR rate was lower than their reported $47 \%$. The lower response rates in our study may reflect a higher proportion of patients with high-risk cytogenetic features ( $56 \%$ vs. $41 \%$ in 
CALGB 9712 and 25\% in the general CLL population) [19]. As well as frequent dose reduction due to toxicity. Additionally, in contrast to CALGB 9712, CT imaging was used to assess response as per the updated 2008 International Working Group response guidelines for CLL [14]. The more sensitive modality of evaluating response may have also contributed to the lower ORR and CR rates in our study. Interestingly, our observations are consistent with those recently presented by Flinn et al., who also reported a comparable ORR of $63 \%$ [13] using the 2008 IWCLL guidelines. Thus, our inability to attain the anticipated $\mathrm{CR}$ rate benchmark compromised our ability to complete the second stage of the study despite the fact that $20 \%$ of the patients when treated with lenalidomide maintenance upgraded their clinical response.

In our study the benefit of lenalidomide was apparent only in patients who were able to achieve an initial response with FR. In two of the three patients who were refractory to FR, lenalidomide was unsuccessful in improving response. The third patient had been removed from study due to rapidly progressive disease, and did not receive lenalidomide. As this study included a small number of patients, limited conclusions regarding the efficacy of sequential lenalidomide in FR-sensitive versus FR-refractory patients should be made. Although lenalidomide failed to induce a response or improvement in response for patients with del $17 p$ or del 11q, the ability of lenalidomide to support the durability of remission in high-risk cytogenetic CLL cases has been demo-nstrated previously [20].

The use of lenalidomide maintenance therapy has been the focus of main upcoming trials. The FRL regimen was studied as part of the North American Intergroup trial 10404 (NCT00602459) in which CLL patients requiring front-line therapy were randomized to FR, FR followed by lenalidomide, or FCR. Patients with del 11q were assigned to the fourth arm of FCR followed by lenalidomide based on previous findings, suggesting better outcomes in this population with the addition of cyclophosphamide. The dosing regimen of fludarabine and lenalidomide were the same as in our study. This trial has completed accrual and results are pending. Other ongoing phase III trials exploring the use of lenalidomide maintenance in CLL include the German CLL Study Group (GCLLSG) CLLM1 study in high-risk patients who have achieved at least a partial response to front-line fludarabine or bendamustine-based induction (NCT01556776), the Australasian Residuum study of lenalidomide after front-line therapy, and the Celgene Corporation-sponsored Continuum study of lenalidomide for patients who have achieved a response to second-line therapy (NCT00774345).

Whether a fludarabine-based regimen remains clinically relevant as a front-line regimen remains controversial. Bendamustine has been approved for previously untreated CLL based on superiority in CR and PFS compared with chlorambucil [21]. Given the promising efficacy and safety data with bendamustine and rituximab (BR) in the front-line setting, the GCLLSG conducted the phase III CLL10 trial of BR versus FCR in previously untreated, physically fit patients [22]. The ORRs with the regimens were identical at $98 \%$, however, both CR rates were suboptimal (FCR 41\%, BR 32\%), with no difference in OS. As the achievement of a complete remission is associated with an overall survival benefit, there is considerable room for improvement with front-line regimens. The availability of novel agents targeting key intracellular signaling pathways downstream of the B-cell has already started to make impact in this disease. Ibrutinib, the first of these small molecules to be approved in CLL, [23] is being investigated not only in combination with FCR (NCT02251548) and BR (NCT01292135) but also in comparison to BR in the upfront phase III Alliance trial of BR vs. R-Ibrutinib vs. Ibrutinib (NCT01886872) and FCR (NCT02048813). Idelalisib, also approved in relapsed CLL, [24] has demonstrated remarkable results in front-line setting when administered with rituximab [25]. Treatments strategies for CLL will continue to evolve dramatically over the next several years. With further investigation, these and newer targeted therapies will likely move to the forefront for CLL to further improve patient outcomes for this disease.

\section{Clinical practice points}

At the time of study conception, fludarabine-based chemoimmunotherapy was the standard of care for previously untreated CLL. While ORRs with these regimens are $90-95 \%$, patients inevitably relapse and further treatment options are necessary. Lenalidomide is a promising agent with activity in CLL. Concurrent FR and lenalidomide is associated with significant toxicity, but this is the first report of lenalidomide as maintenance therapy following FR induction. In this study, the addition of lenalidomide in a sequential manner allowed for better tolerability and less toxicity than prior data with concurrent regimens of FRL. Furthermore, a proportion of patients were able to convert from a partial to complete response. This regimen is also being studied in a four-arm phase II intergroup study evaluating variations of fludarabine-based regimens (FR or FCR) followed by maintenance lenalidomide. Data from this study is not yet available, but may ultimately impact practice guidelines by incorporating the use of maintenance lenalidomide.

\section{Additional files \\ Supplementary Table S1 \\ Supplementary Table S2 \\ Supplementary Data}

\section{Competing interests}

All authors have no competing interests except Dr. Bruce D. Cheson. He has received consulting fees and research support from Teva, Celgene, Pharmacyclics, and Gilead. 
Ujjani et al. Hematology and Leukemia 2015,

http://www.hoajonline.com/journals/pdf/2052-434X-3-2.pdf

Authors' contribution

\begin{tabular}{|l|c|c|c|c|c|c|c|c|c|c|}
\hline Authors' contributions & CU & AP & EG & SJ & TZ & KC & GZ & YA & JC & BDC \\
\hline Research concept and design & -- & $\checkmark$ & -- & $\checkmark$ & -- & -- & -- & -- & -- & $\checkmark$ \\
\hline $\begin{array}{l}\text { Collection and/or assembly } \\
\text { of data }\end{array}$ & $\checkmark$ & $\checkmark$ & -- & -- & -- & $\checkmark$ & -- & $\checkmark$ & $\checkmark$ & -- \\
\hline Data analysis and interpretation & $\checkmark$ & $\checkmark$ & -- & -- & -- & $\checkmark$ & $\checkmark$ & $\checkmark$ & -- & $\checkmark$ \\
\hline Writing the article & $\checkmark$ & $\checkmark$ & -- & -- & -- & -- & -- & -- & -- & $\checkmark$ \\
\hline Critical revision of the article & $\checkmark$ & $\checkmark$ & -- & -- & -- & -- & -- & -- & -- & $\checkmark$ \\
\hline Final approval of article & $\checkmark$ & $\checkmark$ & $\checkmark$ & -- & -- & $\checkmark$ & $\checkmark$ & $\checkmark$ & -- & $\checkmark$ \\
\hline Statistical analysis & -- & -- & $\checkmark$ & -- & $\checkmark$ & $\checkmark$ & $\checkmark$ & $\checkmark$ & -- & -- \\
\hline
\end{tabular}

\section{Acknowledgement}

The conception, design, analysis and interpretation of data from RNA-sequencing experiments were carried out by members of the Asher Chanan-Khan Translational Laboratory in conjunction with the Bioinformatics divisions at Mayo Clinic Florida. We thank Dr. Chanan-Khan for his support in critical review of the data and writing of this manuscript. We would also like to thank Kelly Viola for her editorial assistance.

\section{Publication history}

Editor: Mario Tiribelli, University of Udine, Italy.

EIC: Evangelos Terpos, University of Athens School of Medicine,

Greece.

Received: 27-Mar-2015 Final Revised: 25-Apr-2015

Accepted: 11-May-2015 Published: 18-May-2015

\section{References}

1. Cancer Facts and figures. American Cancer Society. 2015. | Pdf

2. Byrd JC, Peterson BL, Morrison VA, Park K, Jacobson R, Hoke E, Vardiman JW, Rai K, Schiffer CA and Larson RA. Randomized phase 2 study of fludarabine with concurrent versus sequential treatment with rituximab in symptomatic, untreated patients with B-cell chronic lymphocytic leukemia: results from Cancer and Leukemia Group B 9712 (CALGB 9712). Blood. 2003; 101:6-14. | Article | PubMed

3. Woyach JA, Ruppert AS, Heerema NA, Peterson BL, Gribben JG, Morrison VA, Rai KR, Larson RA and Byrd JC. Chemoimmunotherapy with fludarabine and rituximab produces extended overall survival and progression-free survival in chronic lymphocytic leukemia: long-term follow-up of CALGB study 9712. J Clin Oncol. 2011; 29:1349-55. | Article | PubMed Abstract | PubMed Full Text

4. Tam CS, O'Brien S, Wierda W, Kantarjian H, Wen S, Do KA, Thomas DA, Cortes J, Lerner $S$ and Keating MJ. Long-term results of the fludarabine, cyclophosphamide, and rituximab regimen as initial therapy of chronic lymphocytic leukemia. Blood. 2008; 112:975-80. | Article | PubMed Abstract | PubMed Full Text

5. Hallek M, Fischer K, Fingerle-Rowson G, Fink AM, Busch R, Mayer J, Hensel M, Hopfinger G, Hess $G$ and von Grunhagen $U$ et al. Addition of rituximab to fludarabine and cyclophosphamide in patients with chronic lymphocytic leukaemia: a randomised, open-label, phase 3 trial. Lancet. 2010; 376:1164-74. | Article | PubMed

6. Chanan-Khan A, Miller KC, Takeshita K, Koryzna A, Donohue K, Bernstein ZP, Mohr A, Klippenstein D, Wallace P, Zeldis JB, Berger C and Czuczman MS. Results of a phase 1 clinical trial of thalidomide in combination with fludarabine as initial therapy for patients with treatment-requiring chronic lymphocytic leukemia (CLL). Blood. 2005; 106:3348-52. | Article PubMed

7. Chanan-Khan A, Miller KC, Musial L, Lawrence D, Padmanabhan S, Takeshita K, Porter CW, Goodrich DW, Bernstein ZP and Wallace P et al. Clinical efficacy of lenalidomide in patients with relapsed or refractory chronic lymphocytic leukemia: results of a phase II study. I Clin Oncol. 2006; 24:5343-9. | Article | PubMed

8. Sher $\mathrm{T}$, Miller $\mathrm{K}$ and Patel $\mathrm{M}$ et al. Lenalidomide associated tumor flare reaction correlates with clinical response in patients with relapsed or refractory B-cell CLL. Haematologica. 2009; 94:373.

9. Ferrajoli A, Lee BN, Schlette EJ, O’Brien SM, Gao H, Wen S, Wierda
WG, Estrov Z, Faderl S, Cohen EN, Li C, Reuben JM and Keating MJ. Lenalidomide induces complete and partial remissions in patients with relapsed and refractory chronic lymphocytic leukemia. Blood. 2008; 111:5291-7. | Article | PubMed Abstract | PubMed Full Text

10. Brown JR, Abramson J, Hochberg E, Mikler E, Dalton V, Werner L, Reynolds H, Thompson C, McDonough SM, Kuang Y, Ritz J, Neuberg D and Freedman AS. A phase I study of lenalidomide in combination with fludarabine and rituximab in previously untreated CLL/SLL. Leukemia. 2010; 24:1972-5. | Article | PubMed Abstract | PubMed Full Text

11. Egle A, Steurer M and Melchardt T et al. The REVLIRIT CLL5 AGMT Study - a Phase I/II Trial Combining Fludarabine/Rituximab with Escalating Doses of Lenalidomide Followed by Rituximab/Lenalidomide in Untreated Chronic Lymphocytic Leukemia (CLL): Results of a Planned Interim Analysis. Blood (ASH Annual Meeting Abstracts). 2009; 114: 3453. | Article

12. Egle A, Steurer M and Gassner FJ et al. Lenalidomide/Rituximab Maintenance After Induction With Fludarabine/Rituximab In Combination With Escalating Doses Of Lenalidomide In Previously Untreated Chronic Lymphocytic Leukemia (CLL): The Revlirit CLL5 AGMT Phase I/II Study, Final Results. Blood. 2013; 122:21. | Article

13. Flinn IW, Cooper RS, Thompson DS, Waselenko JK, Reeves J and Wise RL et al. Fludarabine, Rituximab, and Lenalidomide in Previously Untreated Patients with Chronic Lymphocytic Leukemia (CLL): A Phase I/II Trial of the Sarah Cannon Research Institute. ASH Annual Meeting Abstracts. 2012; 120:715. | Article

14. Hallek M, Cheson BD, Catovsky D, Caligaris-Cappio F, Dighiero G, Dohner $H$, Hillmen P, Keating MJ, Montserrat E, Rai KR and Kipps TJ. Guidelines for the diagnosis and treatment of chronic lymphocytic leukemia: a report from the International Workshop on Chronic Lymphocytic Leukemia updating the National Cancer Institute-Working Group 1996 guidelines. Blood. 2008; 111:5446-56. | Article | PubMed Abstract | PubMed Full Text

15. Takada K, Zhu D, Bird GH, Sukhdeo K, Zhao JJ, Mani M, Lemieux M, Carrasco DE, Ryan J and Horst D. et al. Targeted disruption of the BCL9/ beta-catenin complex inhibits oncogenic Wnt signaling. Sci Trans/ Med. 2012; 4:148ra117. | Article | PubMed Abstract | PubMed Full Text

16. Zhu N, Gu L, Findley HW, Chen C, Dong JT, Yang L and Zhou M. KLF5 Interacts with p53 in regulating survivin expression in acute lymphoblastic leukemia. J Biol Chem. 2006; 281:14711-8. | Article | PubMed

17. Mittal AK, Hegde GV, Aoun P, Bociek RG, Dave BJ, Joshi AD, Sanger WG, Weisenburger DD and Joshi SS. Molecular basis of aggressive disease in chronic lymphocytic leukemia patients with 11q deletion and trisomy 12 chromosomal abnormalities. Int J Mol Med. 2007; 20:461-9. | Article I PubMed

18. Schweighofer CD, Coombes KR, Barron LL, Diao L, Newman RJ, Ferrajoli A O'Brien S, Wierda WG, Luthra R, Medeiros LJ, Keating MJ and Abruzzo LV. A two-gene signature, SKI and SLAMF1, predicts time-to-treatment in previously untreated patients with chronic lymphocytic leukemia. PLOS One. 2011; 6:e28277. | Article | PubMed Abstract | PubMed Full Text

19. Dohner H, Stilgenbauer S, Benner A, Leupolt E, Krober A, Bullinger L Dohner $\mathrm{K}$, Bentz $\mathrm{M}$ and Lichter $\mathrm{P}$. Genomic aberrations and survival in chronic lymphocytic leukemia. N Engl J Med. 2000; 343:1910-6. | Article I PubMed

20. Sher T, Miller KC, Lee K and Chanan-Khan A. Remission induction with lenalidomide alone in a patient with previously untreated plasmablastic myeloma: a case report. Clin Lymphoma Myeloma. 2009; 9:328-30. | Article | PubMed

21. Knauf WU, Lissichkov T, Aldaoud A, Liberati A, Loscertales J, Herbrecht R, Juliusson G, Postner G, Gercheva L, Goranov S and Becker M et al. Phase III randomized study of bendamustine compared with chlorambucil in previously untreated patients with chronic lymphocytic leukemia. J Clin Oncol. 2009; 27:4378-84. | Article | PubMed

22. Eichhorst $B$, Fink $A$ and Busch $R$ et al. Frontline chemoimmunotherapy with fludarabine, cyclophosphamide, and rituximab (FCR) shows superior efficacy in comparison to bendamustine and rituximab (BR) in previously untreated and physically fit patients with advanced chronic lymphocytic leukemia (CLL): Final analysis of an international, 
Ujjani et al. Hematology and Leukemia 2015,

http://www.hoajonline.com/journals/pdf/2052-434X-3-2.pdf

randomized study of the German CLL Study Group (GCLLSG) (CLL10

Study). Blood (ASH Annual Meeting) 2014; 124: 19. | Article

23. Byrd JC, Furman RR, Coutre SE, Flinn IW, Burger JA, Blum KA, Grant B, Sharman JP, Coleman M and Wierda WG et al. Targeting BTK with ibrutinib in relapsed chronic lymphocytic leukemia. N Engl J Med. 2013; 369:32-42. | Article | PubMed Abstract | PubMed Full Text

24. Furman RR, Sharman JP, Coutre SE, Cheson BD, Pagel JM, Hillmen $P$, Barrientos JC, Zelenetz AD, Kipps TJ and Flinn I et al. Idelalisib and rituximab in relapsed chronic lymphocytic leukemia. $N$ Engl I Med. 2014; 370:997-1007. | Article | PubMed Abstract | PubMed Full Text

25. O'Brien S, Lamanna N and Kipps T et al. Update on a Phase $\mathbf{2}$ Study of Idelalisib in Combination with Rituximab in Treatment-Naïve Patients $\geq 65$ Years with Chronic Lymphocytic Leukemia (CLL) or Small Lymphocytic Lymphoma (SLL). Blood. (ASH Annual Meeting). 2014; 124 1994. | Article

\section{Citation}

Ujjani C, Paulus A, Gehan E, Jamshed S, Zhuang

T, Chitta K, Zhang G, Asmann Y, Crawford J and

Cheson $\mathrm{BD}$. Lenalidomide following fludarabine and rituximab in previously untreated CLL. Hematol Leuk. 2015; 3:2. http://dx.doi.org/10.7243/2052-434X-3-2 